\title{
lothalamate Sodium
}

National Cancer Institute

\section{Source}

National Cancer Institute. Iothalamate Sodium. NCI Thesaurus. Code C47569.

The sodium salt form of iothalamate, an organic iodine compound and a radiographic contrast medium. lothalamate sodium blocks $x$-rays as they pass through the body, thereby allowing body structures not containing iodine to be visualized. The deg ree of opacity produced by iothalamate sodium is directly proportional to the total amount of the iodinated contrast agent in the path of the $x$-rays. The visualization of body structures is dependent upon the distribution and elimination of iothalamate sodium. ( $\mathrm{NCl05)}$ 